\title{
SÍNDROME NEUROLÉPTICO MALIGNO
}

\section{Neuroleptic malignant syndrome}

Fecha de recibido: 13 de abril de 2010 • Fecha de aprobación: 30 de mayo de 2010

Resumen. El síndrome neuroléptico maligno es una emergencia médica rara, grave y potencialmente fatal, que se asocia al uso de medicamentos neurolépticos, cuya prevalencia es de aproximadamente $0,02 \%$ a $3 \%$ de los pacientes tratados, y se caracteriza por presentar hipertermia, rigidez muscular, alteración de la conciencia y disautonomía, además de la elevación de la creatinkinasa (CK), aunque muchas veces no es fácil reconocerlo debido a la variedad de diagnósticos diferenciales. La teoría más aceptada es la relacionada con la actividad dopaminérgica en el Sistema Nervioso Central afectando los núcleos de la base y al hipotálamo, por lo que dentro de su tratamiento se encuentra el uso de agonistas dopaminérgicos tales como la bromocriptina, además del resto de medidas generales. La mortalidad resulta directamente de las manifestaciones disautonómicas de la enfermedad y de las complicaciones sistémicas tales como cardiopatías y falla renal aguda secundaria a rabdomiolisis, si no se trata de manera precoz.

Palabras clave: toxicología, neurolépticos, hipertermia, farmacología.

\begin{abstract}
Neuroleptic malignant syndrome is a rare medical emergency, serious and potentially fatal, associated with the use of neuroleptic drugs, whose prevalence is approximately 0.02 to $3 \%$ of patients, and is characterized by hyperthermia, muscle rigidity, impaired consciousness and dysautonomia, as well as elevated creatine kinase (CK), although often not easy to recognize because of the variety of differential diagnoses. The most accepted theory is related to dopaminergic activity in the central nervous system affecting the basal ganglia and the hypothalamus, so that in its treatment is the use of dopamine agonists such as bromocriptine, in addition to other measures general.
\end{abstract}

Mortality results directly from the dysautonomic manifestations of the disease and from the systemic complications such as heart and acute renal failure secondary to rhabdomyolysis, if not treated early.

Key words: toxicology, neuroleptics, hyperthermia, pharmacology.

\section{INTRODUCCIÓN}

El síndrome neuroléptico maligno es una emergencia médica rara, grave y potencialmente fatal, que se asocia al uso de medicamentos neurolépticos; fue descrita en 1968; caracterizándose por presentar hipertermia, rigidez muscular, alteración de la conciencia y disautonomía.

La mortalidad resulta directamente de las manifestaciones disautonómicas de la enfermedad y de las complicaciones sistémicas, pero ha descendido desde los primeros reportes en la década de los sesenta cuando se estimó que era del 76\%(1).

\section{EPIDEMIOLOGÍA}

La incidencia del síndrome neuroléptico maligno (SNM) es aproximadamente de 0,02 a 3\% de los pacientes tratados con neurolépticos; es mayor en hombres ${ }^{(1)}$. Este síndrome ha sido descrito en todos los grupos etarios, desde los 0,9 hasta los 78 años de edad ${ }^{(2)}$, sin que la edad sea un factor de riesgo ${ }^{(3)}$.

* Hospital Universitario Hernando Moncaleano Perdomo, Neiva, Huila. Correo electrónico: luisalfonso110@hotmail.com 
Aunque los neurolépticos llamados de máxima potencia (haloperidol, flufenazina) han sido los más frecuentemente asociados a la aparición del SNM ${ }^{(4-5)}$, se ha visto que los neurolépticos de baja potencia (ej. clorpromazina) y los llamados fármacos antipsicóticos atípicos (ej. clozapina, risperidona, olanzapina), al igual que los fármacos antieméticos (ej. metoclopramida, prometazina), también pueden producir esta patología ${ }^{(6-7)}$ (Tabla 1).

La aparición de SNM se desarrolla en los siguientes eventos ${ }^{(8)}$.

1. Asociación de varios neurolépticos.

2. Asociación de neurolépticos a antidepresivos tricíclicos o sales de litio.

3. Pacientes con síndromes cerebrales de naturaleza orgánica.

4. Administración parenteral de neurolépticos.

5. Asociación de neurolépticos de acción retardada a agentes antiparkinsonianos.

Tabla 1. Fármacos asociados al síndrome neuroléptico maligno.

\begin{tabular}{|c|c|}
\hline Grupo & Ejemplo \\
\hline Butirofenonas & Haloperidol, bromperidol \\
\hline Fenotiacinas & $\begin{array}{l}\text { Clorpromacina, } \\
\text { levomepromazina, } \\
\text { trifluofenazina, } \\
\text { flufenazina }\end{array}$ \\
\hline Tioxantenos & Tiotixeno \\
\hline Dibenzoxazepina & Loxapina \\
\hline $\begin{array}{l}\text { Agentes depletores } \\
\text { de dopamina }\end{array}$ & $\begin{array}{l}\text { Tetrabenazina, alfa- } \\
\text { metil-tirosina, retirada } \\
\text { de levodopa, levodopa- } \\
\text { carbidopa, amantadina, } \\
\text { metoclopramida }\end{array}$ \\
\hline
\end{tabular}

\section{FISIOPATOLOGÍA}

La causa del SNM es aún desconocida. Las teorías que existen al respecto se basan en las explicaciones de todas las manifestaciones clínicas que se presentan y en la información obtenida.

La teoría más aceptada es la relacionada con la actividad dopaminérgica en el sistema nervioso central que afecta los núcleos de la base y al hipotálamo ${ }^{(9)}$. El bloqueo de los receptores dopaminérgicos a nivel central en el hipotálamo puede causar hipertermia y otros síntomas disautonómicos ${ }^{(10,11)}$. La interferencia con las vías dopaminérgicas nigroestriadas puede causar síntomas de tipo parkinsoniano como rigidez muscular y temblor ${ }^{(11,12)}$. Otros neurotransmisores (GABA, adrenalina, serotonina y acetilcolina) pueden aparecer involucrados de manera directa $\mathrm{o}$ indirecta(13-14).

Las anormalidades motoras son variadas, pero en general son típicas reacciones extrapiramidales parkinsonianas, comúnmente vistas como efectos colaterales de los neurolépticos (Tabla 2). La administración de fármacos tales como la bromocriptina, agonista de la dopamina, disminuye la severidad del $\mathrm{SNM}^{(8)}$.

Una teoría alternativa está relacionada con la rigidez y el daño muscular que se presenta en el sistema muscular, posiblemente por los cambios directos en la función mitocondrial muscular. Esto puede suceder debido a alteraciones primarias en el músculo esquelético o por efectos tóxicos directos originados por neurolépticos en el músculo esquelético ${ }^{(12-15)}$.

\section{MANIFESTACIONES CLÍNICAS}

El SNM se puede definir como la asociación entre un fármaco neuroléptico y la tétrada clínica

Tabla 2. Efectos colaterales de los neurolépticos.

Bloqueo dopaminérgico

Núcleo estriado hipotalámico

Diencéfalo-espinal

S. Reticular Activador Ascendente
Características del síndrome neuroléptico maligno

Rigidez muscular

Generación de calor

Alteraciones en la regulación del tono vascular y de la temperatura

Desregulación autonómica. Rigidez muscular, estupor, mutismo 
como son: fiebre, rigidez muscular, cambios en el estado mental y disautonomía ${ }^{(9-16)}$. Se presentan síntomas piramidales como: hipertonía de predominio axial, llegando incluso al opistótono, postura flexora o extensora y alteración de los reflejos, incluyendo la aparición del reflejo de Babinski. Pueden aparecer síntomas extrapiramidales como: temblor, disartria y disfagia. Se desarrolla compromiso de conciencia, lo más frecuente es obnubilación, confusión y catatonía, el paciente puede eventualmente evolucionar hacia el coma(17). Puede aparecer debido al inicio de la terapia farmacológica o el incremento de la dosis. Las manifestaciones clínicas pueden aparecer en horas o días, estimándose que puede ser entre cuatro y catorce días de iniciado el tratamiento, aunque igualmente puede aparecer en cualquier momento. El 90\% de los pacientes han presentado los síntomas en promedio a los diez días.

\section{DIAGNÓSTICO ${ }^{(18)}$}

El diagnóstico es realizado con base en la historia clínica, un detallado examen físico y hallazgos de laboratorios. Este se puede lograr por medio de los siguientes cinco criterios:

1. Tratamiento reciente con algún neuroléptico generalmente pasadas una a cuatro semanas.

2. Hipertermia (temperatura alrededor de $38^{\circ} \mathrm{C}$, aunque pueden alcanzar temperaturas mayores de $40^{\circ} \mathrm{C}$ ).

3. Rigidez muscular.

4. Cambios en el estado mental

5. Al menos uno de los siguientes síntomas: taquicardia, hipertensión o hipotensión, diaforesis o sialorrea, temblor, incontinencia, incremento de la CK o de los niveles de mioglobina urinaria, leucocitosis; acidosis metabólica.

Además de lo anterior, hay que tener en cuenta lo siguiente:

1. Excluir otras enfermedades neuropsiquiátricas, sistémicas o inducidas por otras drogas.

2. Se puede presentar SNM atípico por lo siguiente:

a. Pueden no estar presentes la rigidez muscular o la hipertermia dentro del cuadro inicial. b. La rigidez muscular y la hipertermia se pueden desarrollar mucho después que los otros síntomas.

c. La presentación atípica del SNM puede deberse al tratamiento con antipsicóticos atípicos.

\section{HALLAZGOS DE LABORATORIOS}

Aunque los datos de laboratorio pueden ayudar a confirmar el diagnóstico de SNM, no existe ningún dato analítico patognomónico de este proceso.

Elevación de la creatinkinasa (CK): es el laboratorio que más se relaciona con esta patología, y se podría pensar que entre más elevada sea la CK más podría ser la severidad de la rigidez muscular, siendo sus valores mayores de 1000 IU/L, incluso alcanzando los $100.000 \mathrm{IU} / \mathrm{L}$. Hay que tener en cuenta que este no es un examen específico debido a que se puede elevar por otras causas, como inyecciones, traumas, etc. Los niveles vuelven a la normalidad una vez se resuelva el SNM ${ }^{(1-2)}$.

Otros laboratorios menos específicos son:

1. Leucocitosis que puede ser entre $10.000 \mathrm{y}$ 40.000 glóbulos blancos $/ \mathrm{mm}^{3(2-6)}$

2. La elevación de la LDH, la fosfatasa alcalina y las transaminasas hepáticas suele ser común.

3. Trastorno electrolítico: hipocalcemia, hipomagnesemia, hipo o hipernatremia, hipercalemia y acidosis metabólica suele ser comúnmente observada.

4. Elevación de mioglobinuria secundaria a rabdomiolisis que conlleva a falla renal $\operatorname{aguda}^{(2)}$.

5. Otros estudios a realizar: gases arteriales, TP, TPT, INR, test para drogas en orina y suero.

Punción lumbar: está indicada en todo paciente con fiebre y alteración mental para descartar meningitis ${ }^{(18)}$.

\section{DIAGNÓSTICO DIFERENCIAL}

1. Síndrome serotoninérgico (que puede aparecer cuando se utilizan inhibidores de la 
monoamino-oxidasa [IMAO] y fluoxetina, o IMAO y L-triptófano, IMAO y tricíclicos) es el diagnóstico relacionado más común, y pueden presentar escalofríos, hiperreflexia, mioclonía y ataxia, además de náuseas, emesis y diarrea. Si se presenta rigidez y diarrea, son menos severos que en el SNM(19-20).

2. Proceso infeccioso del SNC en pacientes en tratamiento neuroléptico, en los cuales se pueden sumar los signos extrapiramidales secundarios a la medicación neuroléptica y los signos de la infección ${ }^{(8)}$.

3. Catatonía letal: puede ser similar al SNM. Los pacientes con este trastorno desarrollan excitación motora, obnubilación, rigidez, fiebre, taquicardia, diaforesis, presión arterial lábil. Si ya se ha comenzado el tratamiento neuroléptico puede ser difícil realizar el diagnóstico diferencial.

4. Golpe de calor: es un trastorno potencialmente mortal que puede verse en pacientes tratados con neurolépticos. Muchos de los síntomas del golpe de calor son comunes con los del SNM, exceptuando que en éste la piel está caliente, seca, no hay sudoración y existe flacidez muscular.

5. Hipertermia maligna: síndrome también potencialmente fatal en el que se produce un hipermetabolismo muscular tras la administración de agentes anestésicos halogenados o relajantes musculares despolarizantes. En la hipertermia maligna (HM) existe rigidez de los músculos esqueléticos e incremento de la temperatura corporal hasta $43^{\circ} \mathrm{C}$.

Existen diferencias entre el SNM y la $\mathrm{HM}^{(8)}$ :

- En la HM existe una alteración muscular evidente.

- La HM es un trastorno genético.

- La HM se asocia al uso de agentes anestésicos.

- La HM se desarrolla en minutos.

- La administración de curare y pancuronio en el SNM produce flacidez.

6. Atropinismo: por sobredosis de anticolinérgicos puede originar somnolencia y elevación de la temperatura. La diaforesis y la inestabilidad autonómica no suelen aparecer.

7. Habría que realizar también diagnóstico diferencial con: la toxicidad por litio, síndro- me anticolinérgico central, estatus epiléptico, encefalopatía tóxica, etc. ${ }^{(8)}$.

\section{TRATAMIENTO}

Lo primero que se debe realizar y lo más importante es suspender de inmediato el agente causal (neuroléptico), incluso también es importante suspender otros agentes psicotrópicos (litio, terapia anticolinérgica, agentes serotoninérgicos). El tratamiento se puede dividir en medidas generales y específicas.

\section{Tratamiento general}

Son todas aquellas medidas que van encaminadas a reducir la morbimortalidad del paciente. A nivel prehospitalario se debe realizar el protocolo de reanimación para evaluar las condiciones clínicas y así encaminar las acciones siguientes.

Dentro de las medidas generales se puede realizar:

1. Suspender cualquier neuroléptico.

2. Monitorizar al paciente, vigilar su condición ventilatoria y cardiaca. Si es necesario, realizar ventilación mecánica, administrar fármacos antiarrítmicos y colocar marcapasos si es necesario, además de requerir manejo médico en unidad de cuidados intensivos.

3. Mantener una hidratación adecuada para evitar hipotensión. Si la CK está muy elevada, se debe realizar hidratación agresiva con alcalinización urinaria para evitar la falla renal secundaria a la alta carga de mioglobina en el riñón debido a la rabdomiolisis ${ }^{(21)}$. El balance hídrico debe ser monitorizado cuidadosamente, manteniendo un flujo urinario mínimo de $50 \mathrm{ml} / \mathrm{hr}^{(17)}$.

4. Bajar la temperatura usando medios físicos como paños fríos. Si es necesario, se pueden realizar medidas más agresivas: lavado gástrico con agua fría, colocar hielo en las axilas. Se puede usar antipiréticos como el acetaminofén.

5. Puede presentarse marcada hipertensión arterial. La clonidina en este caso podría resultar efectiva ${ }^{(22)}$. El nitroprusiato tendría la ventaja de que facilitaría la disminución de la temperatura corporal al producir vasodilatación cutánea ${ }^{(23)}$. 
6. Administrar heparina o heparina de bajo peso molecular para prevenir la trombosis venosa profunda.

7. Se puede usar benzodiacepinas (ej. clonazepam o lorazepam) entre 0,5 y $1 \mathrm{mg}$ en caso de agitación, si es necesario ${ }^{(24)}$.

\section{Tratamiento específico}

Las recomendaciones farmacológicas para el manejo del SNM se basan principalmente en reporte de casos y experiencia clínica, sin que existan estudios clínicos que los recomienden, por lo que a continuación se mencionan los medicamentos que se podrían usar según su mecanismo.

\section{Agonistas dopaminérgicos}

1. Bromocriptina: fuerte agonista de los receptores D2 dopamina y parcial agonista D1. Se administra para restablecer la pérdida dopaminérgica. Dosis: 2,5 mg vía oral (o por sonda nasogástrica, según sea el caso) cada 6 a 8 h sin sobrepasar los $40 \mathrm{mg} /$ día.

2. Amantadina: posee efectos dopaminérgicos y anticolinérgicos y se usa como alternativa a la bromocriptina. Dosis: inicialmente pueden ser $100 \mathrm{mg}$ por vía oral o sonda nasogástrica según sea el caso, llegando máximo a los $200 \mathrm{mg}$ cada $12 \mathrm{~h}$.

3. Levodopa/carbidopa: se ha usado con alguna experiencia exitosa. Dosis: 25/250 mg tres o cuatro veces/día.

Dantrolene: es un relajante de la musculatura esquelética de acción directa (periférica), al producir bloqueo de la liberación de $\mathrm{Ca}+$ por parte del retículo sarcoplásmico de las fibras musculares, evitando así la contracción muscular. Es el tratamiento de elección en la hipertermia maligna. Dosis de 0,25 mg/ $\mathrm{kg}$ hasta $2 \mathrm{mg} / \mathrm{kg}$ vía intravenosa cada 6 o $12 \mathrm{~h}$. Normalmente el rango de la dosis está entre 3 y $5 \mathrm{mg} / \mathrm{kg}$; la dosis máxima es de $10 \mathrm{mg} / \mathrm{kg} /$ día, debido a que una dosis más alta está relacionada con riesgo de hepatotoxicidad(25).

\section{COMPLICACIONES}

La necesidad de un manejo médico oportuno en SNM es esencial e indiscutible. Dentro de las posibles complicaciones tenemos las siguientes:
Deshidratación; trastorno electrolítico; insuficiencia renal aguda secundaria a la rabdomiolisis y a la mioglobinuria; arritmias cardiacas incluidas Torsade de pointes y paro cardiaco; infarto de miocardio; cardiomiopatía; falla respiratoria aguda secundaria a neumonía, embolismo pulmonar, broncoaspiración, SDRA, neumotórax o neumomediastino; trombosis venosa profunda; trombocitopenia; coagulación vascular diseminada; tromboflebitis; falla hepática; sepsis $^{(21-26)}$.

\section{CONCLUSIONES}

El SNM es una entidad que a pesar de ser poco frecuente, debe estar siempre en nuestra mente cada vez que tengamos en frente un paciente bien sea que se le vaya a iniciar tratamiento con neurolépticos o bien sea que ya los venga consumiendo. Se debe realizar una historia clínica muy detallada para reconocer los síntomas, realizar adecuado diagnóstico descartando los diferenciales e inmediatamente suspender los neurolépticos, además de realizar el resto de tratamiento ya descrito. Las recidivas se pueden presentar por la instauración precoz de los neurolépticos, por lo que la mayoría de los autores consideran prudente esperar por lo menos dos semanas. El uso de neurolépticos de baja potencia, a dosis pequeñas e incluso asociados a medicación antiparkinsoniana puede prevenir este síndrome. Se espera a futuro estudios bien diseñados que establezcan un mejor tratamiento para esta patología.

\section{REFERENCIAS}

1. Shalev A, Hermesh H, Munitz H. Mortality from neuroleptic malignant syndrome. Journal of Clinical Psychiatry 1989;50:18.

2. Levenson JL. Neuroleptic malignant syndrome. American Journal of Psychiatry 1985;142:1137.

3. Keck PE Jr, Pope HG Jr, Cohen BM, et al. Risk factors for neuroleptic malignant syndrome. A case-control study. Archives of General Psychiatry 1989;46:914.

4. Chandran GJ, Mikler JR, Keegan DL. Neuroleptic malignant syndrome: case report and discussion. Canadian Medical Association Journal 2003;169:439.

5. Seitz DP, Gill SS. Neuroleptic malignant syndrome complicating antipsychotic treat- 
ment of delirium or agitation in medical and surgical patients: case reports and a review of the literature. Psychosomatics 2009;50:8.

6. Caroff SN, Mann SC. Neuroleptic malignant syndrome. Medical Clinics of North America 1993;77:185.

7. Kogoj A, Velikonja I. Olanzapine induced neuroleptic malignant syndrome-a case review. Human Psychopharmacology 2003; 18:301.

8. García P, Perales S, Ruiz-Cabello M. Síndrome neuroléptico maligno, síndromes hipertérmicos. En: Principios de Urgencias, Emergencias y Cuidado Crítico. http:// tratado.uninet.edu/c090306.html. Capítulo 9.3. Tema 6.

9. Velamoor VR, et al. Management of suspected neuroleptic malignat syndrome. Canadian Journal of Psychiatry 1995;40(9):545-550.

10. Boulant JA. Role of the preoptic-anterior hypothalamus in thermoregulation and fever. Clinical Infectious Diseases 2000;31Suppl 5:S157.

11. Henderson VW, Wooten GF. Neuroleptic malignant syndrome: a pathogenetic role for dopamine receptor blockade? Neurology $1981 ; 31: 132$.

12. Adnet P, Lestavel P, Krivosic-Horber R. Neuroleptic malignant syndrome. British Journal of Anaesthesia 2000;85:129.

13. Heat-exposed rabbits with haloperidol and atropine administration exhibit increased muscle activity, hyperthermia, and high serum creatine phosphokinase level. Brain Research 1996;743:263.

14. Spivak B, Maline DI, Vered Y, et al. Prospective evaluation of circulatory levels of catecholamines and serotonin in neuroleptic malignant syndrome. Acta Psychiatrica Scandinavica 2000;102:226.

15. Gurrera RJ. Is neuroleptic malignant syndrome a neurogenic form of malignant hyperthermia? Clinical Neuropharmacology 2002;25:183.

16. Waldorf S. AANA journal course. Update for nurse anesthetists. Neuroleptic malignant syndrome. AANA Journal 2003;71:389.

17. Faúndez L, Fadic R. Síndrome Neuroléptico Maligno. En: http://escuela.med.puc.cl/ publ/cuadernos/2004/Neuroleptico.html

18. Benzer T. Neuroleptic Malignant Syndrome. En: http:/ / emedicine.medscape. com/ article/816018-overview. 2010.

19. Carbone JR. The neuroleptic malignant and serotonin syndromes. Emergency Medicine Clinics of North America 2000;18:317.

20. Lejoyeux M, Fineyre F, Ades J. The serotonin syndrome. American Journal of Psychiatry 1992;149:1410.

21. Lappa A, Podesta M, Capelli O, et al. Successful treatment of a complicated case of neuroleptic malignant syndrome. Intensive Care Medicine 2002;28:976.

22. Gregorakos L, Thomaides T, Stratouli S, Sakayanni E. The use of clonidine in the management of autonomic overactivity in neuroleptic malignant syndrome. Clinical Autonomic Research 2000;10:193.

23. Blue MG, Schneider SM, Noro S, Fraley DS. Successful treatment of neuroleptic malignant syndrome with sodium nitroprusside. Annals of Internal Medicine 1986;104:56.

24. Caroff SN, Mann SC, Keck PE Jr. Specific treatment of the neuroleptic malignant syndrome. Biological Psychiatry 1998;44:378.

25. Bond WS. Detection and management of the neuroleptic malignant syndrome. Clinical Pharmacology 1984;3:302.

26. Prevention and Treatment of Heat Injury. Medical Letter on Drugs and Therapeutics Journal 2003;45:58. 